\title{
The Centroid Solid Angle and Probability Models of Square Prism Dice Rolls
}

\author{
Paul R. Hurst, Vanessa Tandiman, \\ Brigham Young University-Hawaii \\ BYU-Hawaii \# 1854, 55-220 Kulanui St, Laie, Hawaii 96762-1294 \\ USA
}

Received: July 7, 2020. Revised: November 13, 2020, Accepted: November 14, 2020. Published: November 16, 2020.

\begin{abstract}
Past studies have indicated that the centroid solid angle is related to probabilities of square prism dice rolls. We explain how it is relevant to these probabilities and how to use the spherical projection to calculate the centroid solid angles for the faces on a square prism. These values are then used in a statistical analysis in the quest of constructing a mathematical probability model. The proposed model is based on the principle that the probability of ending up on a particular resting aspect is proportional to the centroid solid angle of that aspect and inversely proportional to a power of the centroid height in that aspect. Using a power of 2.427, this proposed model fits our data of over 60,000 non-symmetrical square prism dice rolls of various sizes (unequal heights and widths) with the largest magnitude Z-score of 1.01. Different powers can potentially describe other situations; e.g. different surfaces, larger dice, heavier dice, etc.
\end{abstract}

Keywords- Centroid Solid Angle, Dice Probability Model, Square Prism Dice, Unfair Dice.

\section{INTRODUCTION}

D $\mathrm{CE}$ rolling has appeared among the early works that lay the foundation of probability theory. The dice in that context typically have the same height and width (the standard six sided die), which is widely regarded as fair and symmetric. For more information on fair dice, see 11. What about dice whose width and height are not equal? What do we know about probabilities involving non-symmetrical objects? [7] discussed the problem of tossing a coin onto a substance that, on contact, immediately dissipates all kinetic energy of the coin. The proposed probabilities in the solution correspond to the ratios of the centroid solid angles, which are calculated by projecting the object's resting aspect onto an enclosing sphere whose center is the centroid and then dividing these surface areas on the sphere by the square of the sphere's radius. There have been a few studies on a related topic, namely the orientation of assembly parts in an automated manufacturing process. 2 and 3 introduced the energy barrier concept and how it is related to the relevant probabilities. If $\mathrm{A}$ and $\mathrm{B}$ are two resting aspects of an object, it could require more energy for the object to transition from A to B than from $\mathrm{B}$ to $\mathrm{A}$. The larger this difference, the more likely the object will favor natural resting aspect $\mathrm{A}$ when tossed onto a surface where the object bounces or rolls. 9 hypothesizes that the probability of a part ending up in a particular resting aspect is directly proportional to that aspect's centroid solid angle and inversely proportional to the height of its center of gravity in that aspect. 4 is a study involving dropping square prism-shaped parts into a vibratory bowl. They propose using the height of the center of mass in a particular resting aspect to adjust the centroid solid angle and obtain what they call the critical solid angle, which represents the corresponding probability of that resting aspect.

We set out to find a probability model that describes dropping dice onto a hard surface, such as a table. All of the relevant work on this problem is relatively old. However, because of 3D printing, robotics, and image recognition, this type of study can be more easily and consistently performed.

We used a 3D printer to make different sizes of dice and we built a dice rolling machine. This allowed us to obtain a relatively large amount of data. Our data did not match up with any of these former studies. Perhaps this is because our situation is different. However, we hypothesized that the probability model would depend on the centroid solid angle and the height of the center of mass.

\section{Centroid Solid Angles of Square Prism DiCE}

\section{A. Square Prism Dice}

The square prism is a rectangular solid with two opposing faces being squares. The length of an edge of either square will be referred to as the width and the length of an edge not associated with a square will be 
referred to as the height. The two square faces will be referred to as bases while the four remaining rectangular non-square faces will be referred to as sides. Each of these six faces corresponds to the six resting aspects of the die on a horizontal surface. If the height is not equal to the width, then the die is not symmetric and not fair.

\section{B. Projecting the Die onto the Surface of a Sphere}
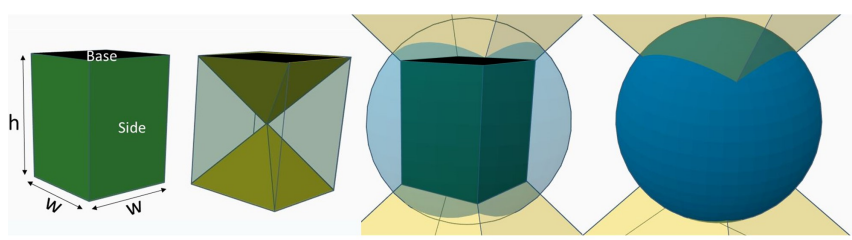

Fig. 1: Left to Right. A square prism, transparent to show planes from the center of mass to the base edges, the sphere segmented by the planes, and the solid sphere segmented by those planes.

Other mathematicians have projected thick coins and other objects onto sphere surfaces. For examples, see references [2], 6], 7], and [8]. Projecting a square prism onto a sphere can be visualized in figure 11. Consider a square prism of uniform density. Construct a sphere that encloses the entire square prism and whose center is at the center of mass of the square prism. Consider a random point on the surface of the sphere. With probability 1 , the radius of the sphere through that point will intersect a face of the square prism. The final resting aspect of the square prism corresponds to that face. If we were tossing the square prism onto a soft sticky surface, one could make a strong case that the probabilities of the square prism landing on various sides are in proportion to the corresponding projected areas (centroid solid angles) on the surface of the sphere. Of course, this is not the scenario we are after, since we are interested in dropping a die onto a hard surface.

\section{B..1 Projected Surface Areas}

Projecting out from the centroid through each edge of a face on the square prism gives us a rectangular region on the surface of the sphere corresponding to that face. This spherical rectangle's area can be calculated from the angles between the geodesics; see figure 2. By Girard's Theorem, if the sphere has radius $R$ the surface area bounded by four geodesics is:

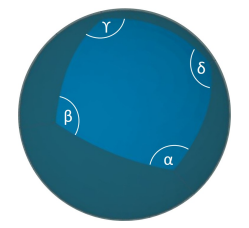

Fig. 2: Spherical rectangle bounded by four geodesics

$$
A=R^{2}(\alpha+\beta+\gamma+\delta-2 \pi)
$$

In the case where $R=1, A$ is the centroid solid angle (CSA) corresponding to that face. Once the spherical rectangle area corresponding to the two bases of the square prism is found from equation (1), the remaining surface area of the sphere corresponds to the sides of the square prism.

\section{B..2 Angles Between the Planes Corresponding to the Bases}

Let the Centroid, O, be positioned at $(0,0,0)$ in the $x y z$-coordinate plane. Let $w$ represent the width, and $h$ represent the height of the square prism. The vertices of the square prism in the coordinate plane

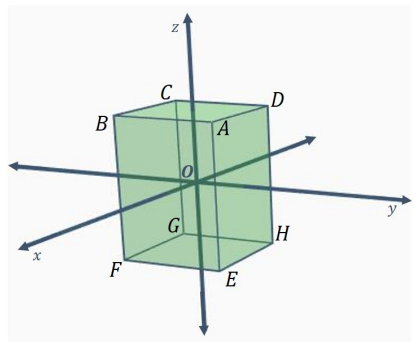
are as follows:

\begin{tabular}{|c|c|c|c|}
\hline Vertex & $x$-coordinate & $y$-coordinate & $z$-coordinate \\
\hline$A$ & $w / 2$ & $w / 2$ & $h / 2$ \\
$B$ & $w / 2$ & $-w / 2$ & $h / 2$ \\
$C$ & $-w / 2$ & $-w / 2$ & $h / 2$ \\
$D$ & $-w / 2$ & $w / 2$ & $h / 2$ \\
$E$ & $w / 2$ & $w / 2$ & $-h / 2$ \\
$F$ & $w / 2$ & $-w / 2$ & $-h / 2$ \\
$G$ & $-w / 2$ & $-w / 2$ & $-h / 2$ \\
$H$ & $-w / 2$ & $w / 2$ & $-h / 2$ \\
\hline
\end{tabular}

Lemma 1. Let $w$ and $h$ represent, respectively, the width and height of a square prism. The angle between the planes from the centroid out through consecutive base edges is

$$
\cos ^{-1}\left(-\frac{w^{2}}{h^{2}+w^{2}}\right)
$$

Proof. For our $A B C D E F G H$ square prism, let the plane $A O B$ be the plane projecting from the sphere's centroid, $O$, to the edge $A B$ of the square prism. The vector originating from $O$ to $A$, called $\mathbf{A}$, and the vector from $O$ to $B$, called $\mathbf{B}$, both lie in the plane $A O B$. Since $O$ lies at the origin, we have:

$$
\mathbf{A}=\left\langle\frac{w}{2}, \frac{w}{2}, \frac{h}{2}\right\rangle \quad \text { and } \quad \mathbf{B}=\left\langle\frac{w}{2},-\frac{w}{2}, \frac{h}{2}\right\rangle
$$

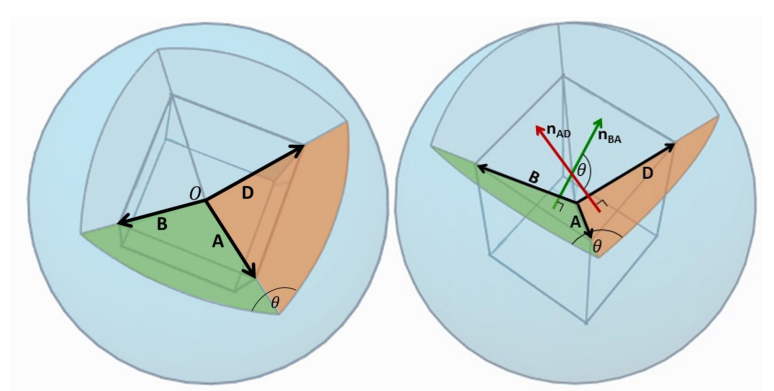

Fig. 3: Adjacent planes from $O$ through base vertices on the base.

The normal vector of the plane $A O B$, called $\mathbf{n}_{B A}$ is obtained from the cross product of $\mathbf{A}$ and $\mathbf{B}$ as follows:

$$
\begin{aligned}
& \qquad \mathbf{n}_{B A}=\mathbf{A} \times \mathbf{B}=\left\langle\frac{w h}{2}, 0,-\frac{w^{2}}{2}\right\rangle \\
& \text { with length }\left\|\mathbf{n}_{B A}\right\|_{2}=\frac{w}{2} \sqrt{h^{2}+w^{2}}
\end{aligned}
$$


Similarly,

$$
\begin{aligned}
& \qquad \mathbf{n}_{A D}=\mathbf{A} \times \mathbf{D}=\left\langle 0,-\frac{w h}{2}, \frac{w^{2}}{2}\right\rangle \\
& \text { with length } \quad\left\|\mathbf{n}_{A D}\right\|_{2}=\frac{w}{2} \sqrt{h^{2}+w^{2}}
\end{aligned}
$$

Let $\theta$ be the angle between the planes $A O B$ and $A O D$, which is the angle between the corresponding geodesics on the surface of the sphere. Using the two normal vectors, $\mathbf{n}_{B A}$ and $\mathbf{n}_{A D}$, we can calculate $\theta$ as follows.

$$
\begin{aligned}
\theta & =\cos ^{-1}\left(\frac{\mathbf{n}_{B A} \cdot \mathbf{n}_{A D}}{\left\|\mathbf{n}_{B A}\right\|_{2}\left\|\mathbf{n}_{A D}\right\|_{2}}\right) \\
& =\cos ^{-1}\left(-\frac{w^{2}}{h^{2}+w^{2}}\right)
\end{aligned}
$$

By symmetry, the other three angles of the spherical rectangle corresponding to the base of the square prism are also equal to $\theta$.

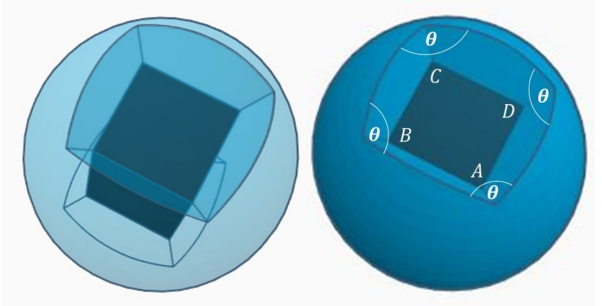

Fig. 4: Transparent and cutout spheres showing the enclosed square prism with planes from the centroid through the top edges of the prism.

\section{Theoretical Probability Calculation using Cen- troid Solid Angles}

Theorem 2. The ratio of the centroid solid angles corresponding to the bases of the square prism to the total centroid solid angle of the entire enclosing sphere is

$$
W_{B}=\frac{2 \cos ^{-1}\left(-\frac{w^{2}}{h^{2}+w^{2}}\right)-\pi}{\pi}
$$

where $w$ and $h$ represent, respectively, the width and height of the square prism.

Proof. Using equation (1) from Girard's Theorem and the value of $\theta$ from Lemma 1 , we calculate the area, $A_{B}$, of the spherical rectangle that corresponds to a base of the square prism:

$$
A_{B}=R^{2}(4 \theta-2 \pi)=R^{2}\left(4 \cos ^{-1}\left(-\frac{w^{2}}{h^{2}+w^{2}}\right)-2 \pi\right)
$$

There are two bases, and the surface area of sphere is
$4 \pi R^{2}$. Thus, the ratio is

$$
\begin{aligned}
\frac{2 A_{B}}{4 \pi R^{2}} & =\frac{2 R^{2}\left(4 \cos ^{-1}\left(-\frac{w^{2}}{h^{2}+w^{2}}\right)-2 \pi\right)}{4 \pi R^{2}} \\
& =\frac{2 \cos ^{-1}\left(-\frac{w^{2}}{h^{2}+w^{2}}\right)-\pi}{\pi}
\end{aligned}
$$

If a point on the enclosing sphere is randomly chosen, the probability that this point is projected out from a point on one of the bases is

$$
W_{B}=\frac{2 \cos ^{-1}\left(-\frac{w^{2}}{h^{2}+w^{2}}\right)-\pi}{\pi}
$$

Since the point corresponding to a base or a side are two complementary events, the probability that the point corresponds to a side is:

$$
\begin{aligned}
W_{S} & =1-W_{B} \\
& =1-\frac{2 \cos ^{-1}\left(-\frac{w^{2}}{h^{2}+w^{2}}\right)-\pi}{\pi} \\
& =\frac{2 \pi-2 \cos ^{-1}\left(-\frac{w^{2}}{h^{2}+w^{2}}\right)}{\pi}
\end{aligned}
$$

III. Statistical Analysis to Evaluate the Use of Centroid Solid Angles in Probability

Models for Square Prism Dice Rolls

As mentioned earlier, the centroid colid angle corresponding to a particular resting aspect of a square prism die seem to affect the probability of the die landing on that aspect. To evaluate how the centroid solid angles can be used in the probability model, we generate experimental data of square prism dice rolls and compare how probability models using centroid solid angles fit the data.

\section{A. Data Generation Methodology}
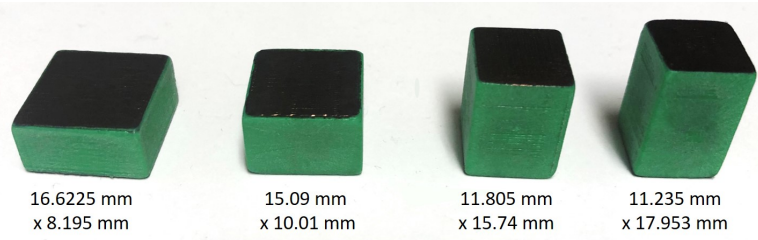

Fig. 5: Four different sizes of square prism dice

For variety, four different sizes of dice were made using a 3D printer. All sizes have roughly the same volume as each other. By adjusting the printer settings, the dice were made to be solid and of uniform density 1 See figure 5. We printed four dice of each size. The actual

\footnotetext{
${ }^{1}$ Dice were printed in PLA using a $5^{\text {th }}$ generation Makerbot ${ }^{\mathrm{TM}}$ Replicator + with $100 \%$ infill density and the floor set higher than the full height of the dice.
} 
Table 1: Observed Data from the Dice Rolling Machine

\begin{tabular}{|l|c|c|c|c|c|c|c|c|}
\hline & $\begin{array}{c}\text { Width } \\
(\mathbf{m m})\end{array}$ & $\begin{array}{c}\text { Height } \\
(\mathbf{m m})\end{array}$ & $\begin{array}{c}\text { Volume } \\
\left(\mathbf{m m}^{3}\right)\end{array}$ & $\begin{array}{c}\text { Ratio } \\
w / h\end{array}$ & $\begin{array}{c}\text { Base } \\
\text { Count }\end{array}$ & $n$ & $\begin{array}{c}\text { Base } \\
\text { Prop. }(\hat{p})\end{array}$ & $\begin{array}{c}\mathbf{9 5 \%} \\
\text { Margin } \\
\text { Error }\end{array}$ \\
\hline A & 16.6225 & 8.195 & 2264 & 2.028 & 13881 & 15593 & 0.89021 & 0.0049 \\
B & 15.09 & 10.01 & 2279 & 1.507 & 11382 & 15756 & 0.72239 & 0.0070 \\
Fair & 13.1 & 13.1 & 2248 & 1 & $\infty$ & $\infty$ & $0 . \overline{3}$ & 0 \\
C & 11.805 & 15.74 & 2193 & 0.75 & 1976 & 14633 & 0.13504 & 0.0055 \\
D & 11.235 & 17.953 & 2266 & 0.62582 & 939 & 14571 & 0.06444 & 0.0040 \\
\hline
\end{tabular}

dimensions of the printed dice varied slightly from the intended dimensions. However, the four dice of each size were identical to each other. To get more accurate measurements of the true dimensions, we placed the four dice consecutively, took total measurements and divided by four.

Dice are typically used in games. Multiple dice are rolled simultaneously onto a hard surface, such as a table top. The dice bounce and spin before coming to a rest. We decided to mimic this situation. A machine was built to automate the process. The dice fall through a series of ramps, similar to what is inside dice rolling towers. The dice then land on a hard surface ${ }^{2}$ We had the machine roll two or three dice at a time. After the dice come to a rest, a picture is taken. See the footnotes for a link to a video of the process ${ }^{3}$

\section{B. Data}

We obtained about 15,000 results for each dice size ${ }^{4}$ The results were recorded and are given in Table 1. Margins of error for $95 \%$ confidence level are given. The column ' $n$ ' is the number of rolls for each size. In addition to the 4 sizes of custom printed dice, the third row represents fair dice, where all sides are equally likely.

\section{Conditions for an Acceptable Probability Model}

As we analyzed models to fit the data for the four dice sizes, we considered the following conditions:

1) When the height and the width of the die are equal, the probability of base is $0 . \overline{3}$. This represents the fair die.

2) Since the number of rolls for each dice size is considerably large, we can use the Normal approximation $N \sim\left(p_{0}, \frac{p_{0}\left(1-p_{0}\right)}{n}\right)$ to evaluate how well the model fits the data for each dice size, where $p_{0}$ is the predicted proportion of bases from the model being evaluated, and $\frac{p_{0}\left(1-p_{0}\right)}{n}$ is the square of the standard error. Because we have four dice sizes, we would have four comparisons, and according to the Bonferroni correction, to get the overall significance level of $\alpha=0.05$, an adjusted $\alpha=\frac{0.05}{4}=0.0125$ is applied for each comparison. This means that

\footnotetext{
${ }^{2}$ All Purpose White Shelf bought from Ace Hardware

${ }^{3} \mathrm{~A}$ video of the machine in action can be found at www.youtube.com/watch?v=OIzivrHaPfY\&

${ }^{4}$ Images of the sample rolls can be found here
}

the Z-scores for all four comparisons should satisfy $-2.50 \leq Z \leq 2.50$.

D. Comparison with Centroid Solid Angle Ratios

As seen in the following table, the data is significantly different than the centroid solid angle ratios calculated from equation (2). The largest $Z$-score $\left(Z=\frac{\hat{p}-p_{0}}{\sqrt{\frac{p_{0}\left(1-p_{0}\right)}{n}}}\right)$ is greater than 75 , which indicates that if the model were correct, then the chance of getting a result at least as extreme as that has a probability of less than $10^{-300}$. Furthermore, none of the Z-scores satisfy the second condition for an acceptable model, where we needed ALL of them to satisfy this condition. Thus, we can safely conclude that simply using the ratios of the centroid solid angles does not give us the correct probabilities. We can see that when the width is greater than the height, the proportion of bases in the sample is significantly higher than the centroid solid angle ratio. Conversely, when the width is less than the height, the proportion of bases in the sample is significantly lower.

Table 2: Comparison of the CSA Model $\left(p_{0}\right)$ and the Observed Data $(\hat{p})$ for the Proportion of Bases

\begin{tabular}{|c|c|c|c|c|}
\hline & $\begin{array}{c}p_{0} \\
\text { (CSA) }\end{array}$ & $\hat{p}$ & $\begin{array}{c}\text { Standard } \\
\text { Devia- } \\
\text { tion }\end{array}$ & $Z$-score \\
\hline A & 0.5951 & 0.8902 & 0.00393 & 75.07 \\
B & 0.4887 & 0.7224 & 0.00398 & 58.69 \\
Fair & 0.3333 & 0 & 0.00385 & 0 \\
C & 0.2344 & 0.1350 & 0.00350 & -28.38 \\
D & 0.1816 & 0.0644 & 0.00319 & -36.69 \\
\hline
\end{tabular}

We suspect that if the dice had been thrown onto an absorbent surface, such as sand, or a sticky surface, then the results would have been significantly closer. The significant difference is due to the energy barrier concept, which most research on this topic mentions. Refer to 2 and 3]. As a die bounces on a hard surface, the amount of kinetic energy is decreasing. Consider a die with $h<w$ in such a situation. If the die has the base facing down, its centroid is lower than if it has one of the sides facing down. Thus, it requires more energy for the die to transition from having the base down to having a side down than is required to flip the other way. As the 
kinetic energy decreases, there is a window where the die has enough energy to transition from side down to base down, but doesn't have enough energy to transition from base down to side down. This accounts for the difference between the theoretical and empirical.

\section{A Model Based on the Centroid Solid Angle And Height that Fits the Data}

\section{A. Modifications Based on Centroid Height}

Aspects that have higher centroid height have more potential energy than aspects with lower centroid height. This indicates that an adjustment similar to the Boltzmann factor can help describe the situation better. Other researchers have also proposed that the probabilities are also related to centroid height [5]. Based on the assumption that the probability of resting in a particular aspect is directly proportional to the centroid solid angle and inversely proportional to the height of the centroid, [9] proposed a method for calculating the probabilities. It gives probabilities equivalent to the following formula:

$$
P(\text { base })=\frac{W_{B} / h_{B}}{W_{B} / h_{B}+W_{S} / h_{S}}, P(\text { side })=1-P(\text { base })
$$

where $h_{B}$ and $h_{S}$ are the heights of the centroids in the corresponding resting aspects, $W_{B}$ is as derived in equation (2) and $W_{S}=1-W_{B}$. As seen in the following table, the probabilities are all closer than the centroid solid angle ratios. However, the largest Z-score is over 40, and none of the Z-scores satisfy the second condition for an acceptable model. We can safely conclude this method does not fit our situation of rolling dice on a hard surface.

Table 3: Comparison of the modified CSA Model $\left(p_{0}\right)$ and the Observed Data $(\hat{p})$ for the Proportion of Bases

\begin{tabular}{|c|c|c|c|c|}
\hline & $\begin{array}{c}p_{0} \\
(\mathbf{C S A} / h)\end{array}$ & $\hat{p}$ & $\begin{array}{c}\text { Standard } \\
\text { Devia- } \\
\text { tion }\end{array}$ & $Z$-score \\
\hline A & 0.7488 & 0.8902 & 0.00347 & 40.71 \\
B & 0.5903 & 0.7224 & 0.00392 & 33.72 \\
Fair & 0.3333 & 0.3333 & $\mathrm{~N} / \mathrm{A}$ & 0 \\
C & 0.1868 & 0.1350 & 0.00322 & -16.06 \\
D & 0.1219 & 0.0644 & 0.00271 & -21.21 \\
\hline
\end{tabular}

We found that if we divide by a power $(p=2.427)$ of the centroid height instead of just the centroid height, the model fits our data better than any other models covered in this paper. Here is the modification;

$$
P(\text { base })=\frac{W_{B} /\left(h_{B}\right)^{2.427}}{W_{B} /\left(h_{B}\right)^{2.427}+W_{S} /\left(h_{S}\right)^{2.427}}
$$

With this modification, the largest Z-score has absolute value 1.01, and all of the Z-scores satisfy the second condition for an acceptable model, as seen in Table 4 and graph. This means that the model could be the correct representation for our situation. In fact, we consider this a spectacular result. Combining geometrical properties with just one parameter, $p$, the model is simultaneously close to all 5 of the data points. Furthermore, by using different values of this one parameter, the model can possibly predict other situations.

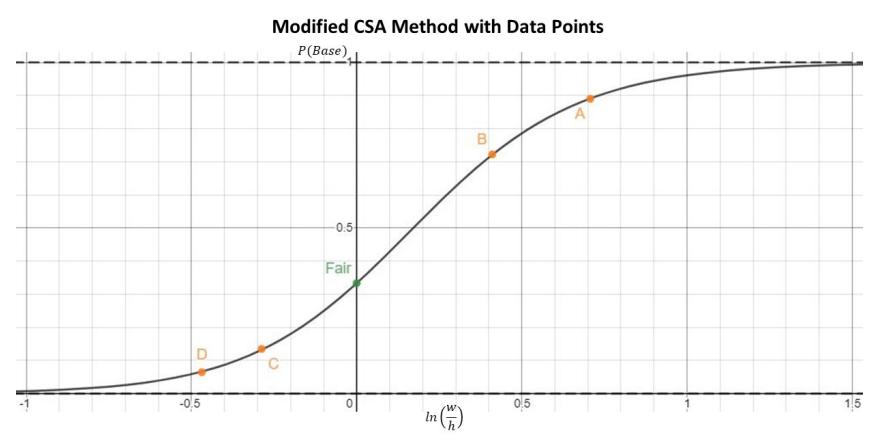

Fig. 6: $\mathrm{P}\left(\right.$ Base $\left.\mid x=\ln \frac{w}{h}\right)=\frac{y-\frac{\pi}{2}}{y-\frac{\pi}{2}+\frac{\pi-y}{e^{2.427 x}}}$

$$
\text { where } \quad y=\cos ^{-1}\left(-\frac{e^{2 x}}{1+e^{2 x}}\right)
$$

Other studies use the ratio $x=\frac{w}{h}$ as the independent variable. However, this yields the domain $(0, \infty)$, with $(0,1)$ representing tall dice and $(1, \infty)$ representing short wide dice. To attain more symmetry, note that we instead use $x=\ln \left(\frac{w}{h}\right)$ as the independent variable. This expands the domain to $\mathbb{R}$ where $(-\infty, 0)$ represents tall dice and $(0, \infty)$ represents short wide dice.

\section{B. Generalized Probability Model using Centroid Solid Angle and Height}

All three previous models can be considered as special cases of this generalized model:

$$
P(\text { base })=\frac{W_{B} /\left(h_{B}\right)^{p}}{W_{B} /\left(h_{B}\right)^{p}+W_{S} /\left(h_{S}\right)^{p}}
$$

Table 4: Comparison Between Modified $\operatorname{CSA}\left(p_{0}\right)$ and Observed Data $(\hat{p})$ with parameter $p=2.427$

\begin{tabular}{|c|c|c|c|c|c|c|c|}
\hline & $\ln \left(\frac{\mathbf{w}}{\mathbf{h}}\right)$ & $n$ & $\begin{array}{c}p_{0} \\
\left(\mathbf{C S A} / h^{2.427}\right)\end{array}$ & $\hat{p}$ & $\begin{array}{c}\text { Residuals } \\
\left(\hat{p}-p_{0}\right)\end{array}$ & $\begin{array}{c}\text { Standard } \\
\text { Deviation }\end{array}$ & $Z$-score \\
\hline A & 0.7072 & 15593 & 0.8911 & 0.8902 & -0.0008 & 0.00250 & -0.34 \\
B & 0.4105 & 15756 & 0.7213 & 0.7224 & 0.0011 & 0.00357 & 0.31 \\
Fair & 0 & $\infty$ & 0.3333 & 0.3333 & 0 & 0.00385 & 0 \\
C & -0.2877 & 14633 & 0.1322 & 0.1350 & 0.0028 & 0.00280 & 1.01 \\
D & -0.4687 & 14571 & 0.0664 & 0.0644 & -0.0020 & 0.00206 & -0.96 \\
\hline
\end{tabular}


$p=0$ represents the centroid solid angle ratio, which has a good chance of working if the die is dropped into sand or onto a sticky surface. $p=1$ represents equation (3), and might work if the die is dropped onto a soft surface. $p=2.427$ works well in our situation of dropping the die onto a hard surface. In general, $p$ is a parameter that depends on how fast the energy of the die dissipates relative to the energy required to change resting aspects. A slower dissipation of energy means that the window where the die has enough energy to go from an aspect with higher centroid height to lower centroid height but can't go from lower to higher will be longer. This means that the die will end up in the aspect with lower centroid height more often. A larger value of $p$ in the model can account for this change. Thus, larger values of $p$ are associated with slower energy dissipation rates and viceversa. The energy dissipation rate depends on various factors, including the hardness, or coefficient of restitution, of the surface and the die. In particular, a larger coefficient of restitution means that less kinetic energy is lost with each bounce of the die, leading to a slower dissipation rate and hence a larger value of $p$. The dissipation rate may also depend on the weight and volume of the dice. By adjusting the power $p$, the model has a chance to work under varying conditions.

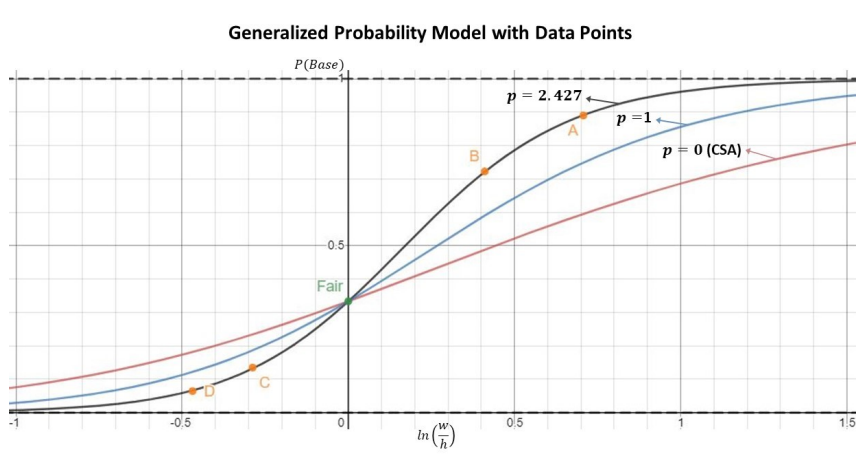

Fig. 7: $\mathrm{P}\left(\right.$ Base $\left.\mid x=\ln \frac{w}{h}\right)=\frac{y-\frac{\pi}{2}}{y-\frac{\pi}{2}+\frac{\pi-y}{e^{p x}}} \quad$ where $y=\cos ^{-1}\left(-\frac{e^{2 x}}{1+e^{2 x}}\right), \quad$ and with $p=0,1,2.427$

\section{A Model BASEd on the Four Parameter} Logistic Function that Fits the DATA

The situation is similar to a logistic function. In particular, the data points fit the following four parameter logistic model fairly well.

$f(x)=1.001-\frac{1.0106}{1+.5157 e^{3.9477 x}} \quad$ where $x=\ln \left(\frac{w}{h}\right)$

As seen in the following table, with this model the largest $Z$-score absolute value is 1.02 . The weighted error summed over the five data points is $\sum_{i=1}^{5} n_{i}\left(\hat{p}_{i}-p_{i}\right)^{2} \approx$ 0.262 .

\begin{tabular}{|c|c|c|c|c|}
\hline & $\begin{array}{c}p_{0} \\
(4 \mathbf{P L})\end{array}$ & $\hat{p}$ & $\begin{array}{c}\text { Standard } \\
\text { Devia- } \\
\text { tion }\end{array}$ & $Z$-score \\
\hline A & 0.8927 & 0.8902 & 0.00248 & -1.02 \\
B & 0.7199 & 0.7224 & 0.00358 & 0.70 \\
Fair & 0.3333 & 0.3333 & 0.00385 & 0 \\
C & 0.1331 & 0.1350 & 0.00281 & 0.69 \\
D & 0.0653 & 0.0644 & 0.00205 & -0.41 \\
\hline
\end{tabular}

The following is a graph of the function with the data points. Of course, we are not allowed to extrapolate beyond where we have data. However, such an extrapolation has a reasonable chance of working. Note that the graph goes above 1 and below 0 . This is seemingly a violation of the laws of probability. However, as the width is increased, the height will eventually become so thin that tiny vibrations on the rolling surface will cause the probability of landing on a base to be 1 . Conversely, as the width is decreased, the probability of landing on the base eventually becomes 0 .

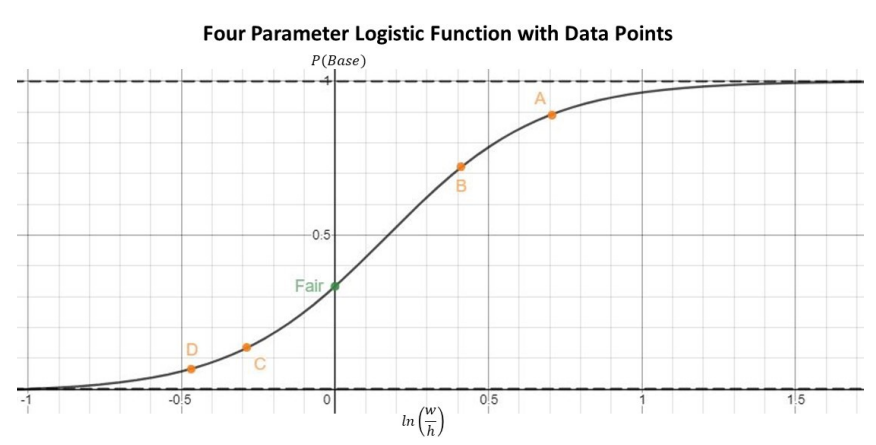

\section{CONCLUSION}

This paper introduces a way to calculate centroid solid angles for square prisms, using only the width and height of the prism. We also propose a non empirical probability model for square prism dice rolls using the centroid solid angle and a power, $p$, of the centroid height. The parameter $p$ depends on the energy dissipation rate as the die bounces. By varying the value of $p$, this model could potentially work for various situations. When $p=2.427$, this theoretical model agrees well with our collection of over 60,000 dice rolls on a hard surface (largest Z-score is 1.01). Before coming up with this model, we experimented with other models that also involved adjustments to the centroid solid angle. However, all previous attempts had to be ruled out because the Z-scores were excessively large in magnitude.

The scenario that we tried to achieve is that of rolling or dropping dice onto a table with a wood or hard laminate surface. Unlike a table, the dice machine has walls around the platform with which the dice collide. It is unclear what effect, if any, this has on the probabilities. The study can be improved by printing out a few other sizes of dice.

Other convex shapes of dice should be tested. Suggested shapes include right circular cylinders of varying thicknesses (thick coins), general rectangular solids, 
tetrahedra, triangular prisms and other prisms. Larger or smaller sizes should be tested. Perhaps a generalized probability model the covers a significantly larger variety of convex shaped dice can be obtained. Our initial testing indicates that the geometry of the triangular prism and the thick coin have a significant effect on the probabilities, in addition to the effects of the center of mass and centroid solid angle. Since 3D printers can make loaded dice, that is an additional avenue that can be explored.

\section{ACKNOWLEDGMENT}

The authors thank the following individuals for their contributions to the project; Doneliezer Baize, Rulon Olmstead, Kenneth Lee, Sione Fuluvaka, Michael Mendoza, Lok Him Rhine, Bo Liu, and Michael Loran.

\section{REFERENCES}

[1] P. Diaconis and J. B. Keller, "Fair Dice". American Mathematical Monthly 96(4): 1989, pp. 337-39. DOI: $10.2307 / 2324089$

[2] G. Boothroyd, A. H. Redford, C. Poli, and L. E. Murch "Statistical distributions of natural resting aspects of parts for automatic handling." SME Manufacturing Engineering Transactions. 1:72, 1972

[3] G. Boothroyd and C. Ho "Natural Resting Aspects of Parts for Automatic Handling." Journal of Engineering for Industry. Vol. 99, 1977, pp. 314-317. DOI: $10.1115 / 1.3439214$

[4] K. A. Ngoi, S. W. Lye, and J. Chen "Analyzing the Natural Resting Aspect of a Prism on a Hard Surface for Automated Assembly". International Journal of Advanced Manufacturing Technology 11(6): 1996 pp. 406-412. DOI: 10.1007/BF01178966

[5] P. S. K. Chua and M. I. Tay "Modeling the Natural Resting Aspect of Small Regular Shaped Parts". Journal of Manufacturing Science \& Engineering 120(3): 1998, pp. 540-546. DOI: 10.1115/1.2830157

[6] R. Olmstead and D. Baize "Probabilities Involving Standard Trirectangular Tetrahedral Dice Rolls". Rose-Hulman Undergrad. Math. J. 19(1): Art. 4. https://scholar.rosehulman.edu/rhumj/vol19/iss1/4/, 2018

[7] F. Mosteller "Fifty Challenging Problems in Probability with Solutions." Mineola, NY, Dover Publications: 1987 , pp. 58-59.

[8] J. Wiegley, A. Rao, and K. Goldberg "Computing a statistical distribution of stable poses for a polyhedron." Proceedings Of the Annual Allerton Conference on Communication Control and Computing. University of Illinois 30, 1992

[9] B. K. A. Ngoi, S. S. G. Lee, and L. E. N. Lim "Analysing the Probabilities of the Natural Resting Aspects of a Component with a Displaced Centre of Gravity". International Journal of Production Research 33(9): 1995, pp. 2387-2394. DOI: $10.1080 / 00207549508904822$

Both authors worked on everything. This includes 3D designing and printing, analyzing dice images, constructing mathematical models, and writing the paper.

\section{Creative Commons Attribution License 4.0 (Attribution 4.0 International, CC BY 4.0)}

This article is published under the terms of the Creative Commons Attribution License 4.0

https://creativecommons.org/licenses/by/4.0/deed.en_US 